233 10
LA

Intormal Report

MASTER
UC-20

Reporting Date: January 1975

Issued: February 1975

\title{
Toroidal Mode Structure in the Scyllac Full Torus
}

by

F. C. Jahoda

R. Kristal

W. E Quinn

$\div$

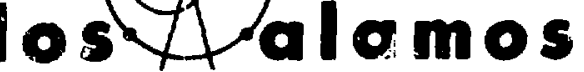

sciontific labioratory

of the University of Californie

LOS ALAMOS, NEW MEXICO 87544

$\downarrow$

An Atfirmative Action/Equal Opporlunity Employer 
In the interest of prompt distribution, this LAMS report was not edited by the Technical Intormation slaff.

Printed in the Uniled States of America Avalable trom National Technical Inlormation Servic*

U.S. Department of Commerce

5285 Port Poyal Rood

Price: Prinled Copy \$4. Y. Microtiche \$2.25

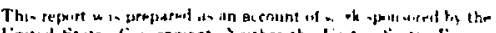

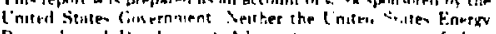

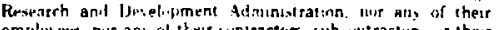

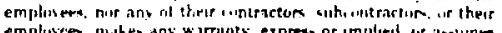

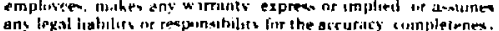

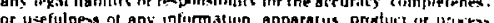

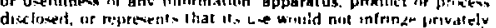
on ned nigh? 
TOROIDAL MODE STRUCTURE IN THE SCYLIAC FULL TORUS

by

F. C. Jahoda, R. Kristal, and W. E. Quinn

ABSTRACT

\begin{abstract}
A $360^{\circ}$ multiple-slit framing camera (11 viewing slits) has shown that the Scyllac toroidal moje structure is consistent in a statistical sense with the $m=1$ instability predictions of MHD sharp-boundary theory. The first few modes of finite wavelength around the torus have growth rates comparable to the $k=0$ mode, and the developed plasma mode structure approaches cut-off at the theoretical value given by magnetic field bending effects. The data indicate that the $m=1$ instability rather than loss of equilibrium terminates the plasma conf inement.
\end{abstract}

\section{INTRODUCTION}

The Scyllac full torus experiment is described in the proceedings of the 5th IAEA Conference on Plasma Physics and Controlled Nuclear Fusion Research, Tokyo, November 11-15, 1974. [1] Measurements of the toroidal mode structure as determined by $\operatorname{six}$ simultaneously acquired data points are reported in Ref. 1. The six points could only determine the amplitude of a constant displacement $(n=0)$ and the amplitudes and phases of the next two harmonics $(n=1,2)$, where $n$ is the axial mode number defined by the plasma displacement $\xi_{2}=\xi_{0} e^{i(n \theta-n z / R)}$, with $\theta$ and $z$ the azimuthal and toroidal coordinates, respectively, and $\mathrm{k}$ the torus major radius. (It is observed that only the $m=1$ mode develops in the minor toroidal cross section. This is attributed to Li:e effects of finite Larmor radius stabilization. [2] ) Statistical tests of the least-squares best $f$ it to the data showed that the plasma dispiacement must consist of more than the $n=0,1$, and 2 harmonics. [1] Randomness of phase between modes causes unreproducibility of the plasma motion from discharge to discharge and requires $(2 n+1)$ simultaneous viewing siots tu reasure $n$ harmonics. We have subsequently measured 11 positions simultaneously, permitting determinations of up to $n=5$. The more definitive mode structure measurements tius achieved are the suhiect of this report.

\section{THEORY}

The theoretical growth rate, $Y$, of the $m=I$ instability is given in sharp boundary theory by [3]

$$
\begin{aligned}
\gamma^{2}(n)= & Y^{2}(0)-v_{A}^{2}(2-\beta) \frac{n^{2}}{R^{2}} \\
\gamma^{2}(0)= & h^{2} v_{A}^{2}\left[-\beta\left(\frac{a}{b}\right)^{4}+\frac{\beta(4-3 \beta)(2-\beta)}{8(1-\beta)} h^{2} a^{2} \delta_{1}^{2}\right. \\
& \left.+\frac{\beta(3-2 \beta)(1-\beta)}{(2-\beta)} \delta_{0}^{2}\right]
\end{aligned}
$$

where $V_{A}$ is the Alfuén velocity, a is the plasma radius, $b$ is the coil radius, $h$ is the wavemumber of the applied $l=l$ and $l=0$ correction fields, $B_{\ell} \sim$ $\sin (Q \theta-h z)$, that establish equilibrium in the tiroidal configuration, $\delta_{1}$ and ${ }^{j} \mathrm{u}$ are the corresponding first-order perturbations of the plasma surface $\mathbf{r}=\mathbf{a}\left[1+\delta_{1} \cos (\theta-h z)-\delta_{0} \cos h z\right]$, and $\dot{\mathrm{j}}$ is the ratio of plasma pressure to the confining magnetic field pressure. 
For the Scyllac parameters ${ }^{11]} \gamma(0)=0.7 \mathrm{MHz}$ and Eq. (1) first becomes negative (the condition for stability) at $n=7$. Furthermore, due to the parabolic dependence on $n, \gamma(n) / \gamma(0)$ is still 0.9 for $n=3$ and only decreases rapidly near the limiting $n$ value.

\section{I. EXPERIMENTAL ARRANGEMENT}

The experimental arrangement is indicated in Eig. 1. A single image converter framing camera located at the geometrical center of the torus has 11 horizontal viewing slits, almost uniformly spread in angle over $360^{\circ}$, imaged on its photocathode. The light from each slit is relayed by two plane mirrors, one directly above the slit, and the second a nember of a multi-facet pyramid structure also centered on the toroidil axis, to a Cassegrainian telescope (fiz.3, focal length $41 \mathrm{~cm}$ ), which images the slits at 10:1 reduction in an approximately circular array onto the $2.5-\mathrm{cm}$ diameter photocathode.

In order to measure the plasma location within each slit, the slit ends had to be well defined in the image. This was accomplished by a separate long time exposure (in the focusing mode of the camera) of reflecting tape on the slit ends illuminated by individual flood lamps.

An example of the raw data is shown on the left ai fig. 2. The circular arrangement is the somewhat arbitrary result of the alignment procedure, and

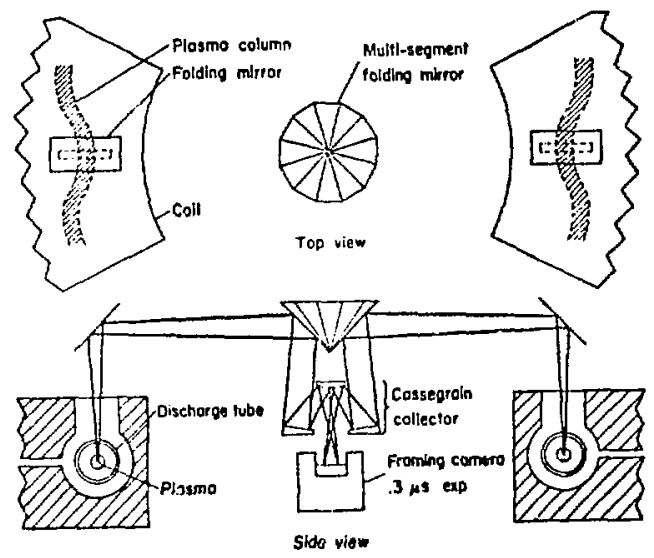

$\because$ ig. 1. $360^{\circ}$ iraming camera arrangement on Scyllac. The plasma is viewed at 11 different circumlurential locations in the toroidal plane. while it evokes a mental image of the toroidal geometry, in actual fact, the images are inverted, so that the ends nearest the center of the image are

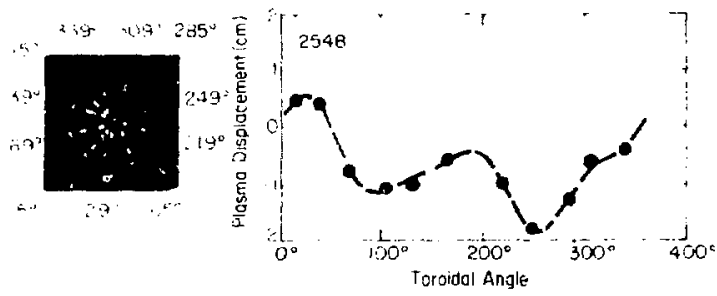

Fig. 2. Framing camera photograph (lef $\tau$ ) and radial plasma displacement vs. toroidal angle witi least-square best fit curve using four harmonics (right).

actually toward this outside, and the large aspect ratio of the torus is complecely distorted.

\section{DATA ANALYSIS}

Numerical values of the plasma displacement from the discharge tube axis at each anguilar position are obtained with the Graf-Pen digitizer under the control of a conputer program which then immediately proceds to a curve fitting routine. The analytical form of the fitting function is :hosen to be

$$
d=a_{0}+\sum_{\ell=1}^{n}\left(a_{\ell} \cos \ell \phi+b_{\ell} \sin \ell \phi\right) .
$$

The inputs are the 11 values of displacement $d$ at the 11 different angular positions $\phi$ and for: a specified $n<5$ thi $(2 n+1)$ a and $b$ coefficlents are determined in a least-square sense with $2(5-n)$ degrees of freedom. The results are displayed in terms of a mode amplitude, $\left(a_{\ell}+b_{\ell}\right)^{1 / 2}$, and a m.ode phase angle, $\tan ^{-1}\left(b_{\ell} / a_{\ell}\right)$. Also computed is the weighted variance of the $f i t$, i.e., the square rost of the sum of the squares of the deviations of the data points from the curve divided by the number of degrees of freedom. Figure 3 illustrates a succes. sion of curves with an increasing number of modes, $n$, fitced to the data of one particular discharge. Over a collection of 15 such discharges, the weighted variance is a minimum either at $n=3$ or at $n=4$, with almost the same number of times for each. 
This means that three modes fit the data pretey we11. with the ath harmonic penerally of smaller amplitude aldiag lie fit only is small amount. On the other hasd, sing only 2 harmonfes, the maximum possible whth the earlier diata from only 6 positions, the fit is definitely worse. It stould also be noced that using 5 hirmonics forces an exact flt to the 11 dalit polnts, and is statistically meaningless since the curve is forced to pasy precisely through the data points, wich themselves are determined with less than absolute precision. It is significant, nonetheless. that when 5 harmonics fare fieced, the amplitude of the 5 th on average is only slightly larger than the 4th, and saller than the first three, in support of the conclusion that there is a rapld fall-off in amplitude with incroasing mode number.

As a consequence of the above results, a fitting procedure with all mode numbers $n=0$ to $n+4$ wals sclected for the best representation of the data. The cabulated chi-square distribution for 2 degrees of freadon fits the distribution obtalned from the sums of squares of the deviations if the expertmental accuracy o is chosen $t a$ be $0.35 \mathrm{~cm}$. Repeated readings of the data findicate that this relatively large uncertilinty is not unreasonable. The phistse angles of the modes appear randorly distributed, and the relative amplleudes also vary from discharge to discharge. In conseq̧uence, the plasma column assumes a different shape every discharpe, als 1llustrated by comparing the 4 harmontes case in fig. 3 with the right side of Fig. 1. This is fust what would be expected for a superposition of several
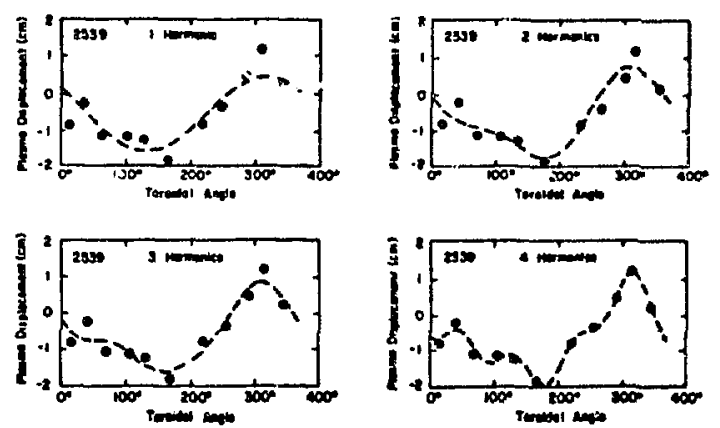

Fig. 3. Radial plasma displacement vs. toroldal angle for one discharge successively fitted with an increasing number of harmonic terns.
Instablity modes, and all iurcher inferences must be of a statistical nature.

The inplitudes of the various rodes, $n=0$ ehrough na4, were averiged over $15 \mathrm{discharges}$ and are plotece in the lower halt of Fig. 4. To compate with theory. we use the calculated n=0 growsh rate of 0.7 miz and the relat lve aprowh rates for the other $n$ numbers, as indlcated by the upper half of Fiff. 4, as well ats the actuit e lme, to 4.6 is, wher: data were vbtained. The relative displacements of the various aodes are then calculated on the "white notse" assumption of equal initial aaplieudes. The absolute scale value is deectrined by ilteing to the obscrved diata point for $n=1$. This sets the coefficient of the growing exponentiat $x=x_{0} e^{\text {it }}$ at $x_{0}=0.023 \mathrm{~cm}$. The resuleant calculated displaremenes are ploted as crosses in ehe lower habf of Fig. 4. and conrected by the soldd line.

The scale is not ficted to nol because it is a soracwat spectal case wilich is only a constan: shift cem in pastetion vs. ankle and can be confused wh small errors in setcing un the correct equilibflum condltions due, for instance, co slightly wrong infetal fill preasure. There should in facs be an infetal 6-the fribard equilibrius silite since all the viewing silts were in troove locations, and using thes or some rether value instead of the discharge

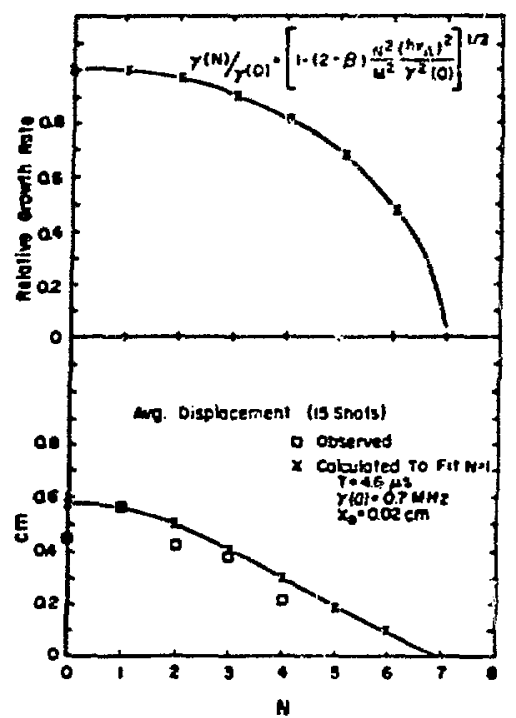

Fig. 4. Spectrum of theorectcal growth raies (upper) and average displatement $v$ s. mode lumber (lower). 
tube center would slightly change the observed value for $n=0$.

\section{v. CONCLUSIONS}

The data presented are entirely consistent with sharp boundary calculations for the expected $m=1$ instability modes. A priori, instability might be expected to develop with equal likelihood in the vertical plane. Streak camera pictures, however indicate much less vertical than horizontal motion. This could well be explained by slight force imbalances in the horizortal plane providing the initial perturbation from which the instability grows primarily in the toroidal plane. These could arise irom small differences in the main magnetic field around the torus. We must then distinguish instability motion resulting from an inftial perturbation due to force imbalance from the total motion being the result of the longer term action of this force imbalance.

Simple arguments can be invoked to show that a force imbalance of only $2 \%$ can establish the initial perturbation of $0.02 \mathrm{~cm}$ in about $0.4 \mu \mathrm{\mu}$, 1.e., a time short compared to when the position determination is made, so that the instability calculation can actually be applied from essentially $t=0$. On the other hand, the same $2 \%$ forre imbalance concinuing to act in the absence of instabllicy would give a trajectory which is too slow to explain the observed linit on plasma confinement. This reinforces our interpretation of the data.
This argument breaks down if the force imbalance is assumed appreciably larger, and the actual value that might have existed is unknown. $\therefore$ significant experiment would have been to measure at two instants of time during the same discharge, to determine whether the separate n modes grow at different rates. Neither the avallable light level nor the exigencies of scheduling permitted such a measurement. It must also be realized that the plasma parameters are continually changing in time, especlally up to peak compression, and we have used a very simple model Independent even of the observed axial flow over the first few microseconds. With these qualifications in mind, the $m=1$ MHD instability theory is the most likely interpretation of the observations.

\section{ACKNOWLEDGMENTS}

We gratefully acknowledge our Indebtedness to the entire Scyllac operating crew and especially thank F. L. Ribe and R. E. Siemon for clarifying discussions.

\section{REFERENCES}

1. E. L. Cantrell, W. R. Ellis, H. W. Harris, F. C. Jahoda, R. Kristal, M. D. Machalek, J. R. McConnell, W. E. Quinn, F. L. Ribe, G. A. Sawyer, F. T. Selbel, and R. E. Siemon, Paper CN-33/E 1-2, Proc, 5th Int. Conf., Tokyo, (1974) IAEA (to be oublished).

2. J. P. Freidberg, Phys. Fluids 15, (1972) 1102 .

3. F. L. Ribe and M. N. Rosenbluth, Phys. Fluids 13. (1970) 2572. 